\title{
SIMULAÇÃO NUMÉRICA DE CRESCIMENTO DE TUMORES MALIGNOS DA PRÓSTATA USANDO A EQUAÇÃO DIFERENCIAL DE GOMPERTZ
}

\author{
CAVALCANTI FILHO, Gentil Fideles ${ }^{1}$ \\ SILVA, Cleomacio Miguel da ${ }^{2}$
}

RESUMO: O câncer de próstata é uma doença que tem atingido a população masculina em todo mundo. Atualmente, diversas técnicas de cirurgia e tratamento foram desenvolvidas para auxiliar no combate ao câncer de próstata. A formação de tumores malignos da próstata ocorre quando as células se dividem rapidamente e de maneira descontrolada, invadindo tecidos e órgãos, e muitas vezes espalhando-se para diferentes regiões do corpo, num processo conhecido como metástase. Entretanto, apesar dos avanços médicos e tecnológicos, o número de óbitos causados pelo câncer de próstata tem aumentado significativamente no Brasil. Para auxiliar no combate ao câncer de próstata, como também entender melhor o comportamento do desenvolvimento tumoral, é necessário conhecer seus mecanismos de crescimento. Para tanto, diversas pesquisas têm sido desenvolvidas com a finalidade de se estudar o crescimento tumoral através de modelos matemáticos, visando obter informações que poderão auxiliar na obtenção de diagnósticos mais precisos, como também verificar em que estágio de desenvolvimento o tumor se encontra, além de outras informações que cada modelo possa fornecer. Sendo assim, dentro desse contexto, o objetivo do presente trabalho foi usar os métodos numéricos de diferenças finitas e Runge-Kutta para simular o crescimento de tumores malignos da próstata, usando a equação diferencial de Gompertz. Os resultados obtidos mostraram que os métodos numéricos de diferenças finitas e Runge-Kutta são ferramentas matemáticas eficientes para simular o crescimento tumoral da próstata.

Palavras-chave: Câncer. Biomatemática. Métodos numéricos.

\section{NUMERIC GROWTH SIMULATION OF MALIGNANT PROSTATE TUMORS USING GOMPERTZ DIFFERENTIAL EQUATION}

\begin{abstract}
SUMMARY: Prostate cancer is a disease that has hit the male population throughout the world. Currently, several techniques of surgery and treatment have been developed to assist in the fight against prostate cancer. The formation of malignant tumors of the prostate occurs when cells divide rapidly and uncontrollably, invading tissues and organs, and often spreading to different regions of the body, in a process known as metastasis. However, despite medical and technological advances, the number of deaths caused by prostate cancer has increased significantly in Brazil. To help in the fight against prostate cancer, as well as better understand the behavior of the tumor development, it is necessary to know its mechanisms of growth. For that, several researches have been developed with the purpose of studying tumor growth through mathematical models, aiming to obtain information that may help in obtaining more accurate diagnoses, as well as to verify at what stage of development the tumor is found, besides other information that each model can provide. Thus, within this context, the objective of the present work was to use the numerical methods of finite differences and Runge-Kutta to simulate the growth of malignant tumors of the prostate, using the Gompertz differential equation. The results showed that numerical methods of finite differences and Runge-Kutta are efficient mathematical tools to simulate tumor growth of the prostate.
\end{abstract}

Keywords: Cancer. Biomathematics. Numerical methods.

\section{INTRODUÇÃO}

O câncer de próstata é uma das doenças que mais mata homens no Brasil (BACELAR JÚNIOR et

\footnotetext{
${ }^{1}$ Escola Politécnica de Pernambuco - Departamento Básico

${ }^{2}$ Universidade de Pernambuco-Campus Mata Norte - Departamento de Biologia
} 
al., 2015). O câncer de próstata é o diagnóstico de câncer mais comum entre os homens. Anualmente, nos Estados Unidos, surge mais de 160 mil novos casos. Embora que, na maioria das vezes, existe quase 100\% de cura quando diagnosticado precocemente, o câncer de próstata continua sendo a terceira principal causa de morte por câncer em homens (LITWIN; TAN, 2017). Quando se suspeita de câncer de próstata, a biópsia de tecido continua sendo o padrão de atendimento para o diagnóstico. No entanto, a identificação e caracterização da doença têm se tornado cada vez mais precisas através de uma melhor estratificação de risco e avanços na ressonância magnética e na imagem funcional, bem como a partir do surgimento de biomarcadores. Atualmente, existem várias opções de tratamento para homens diagnosticados com câncer de próstata (LITWIN; TAN, 2017). A vigilância ativa (a monitorização em série para a progressão da doença com a intenção de curar) parece ser segura e se tornou a abordagem preferida para homens com câncer de próstata menos agressivo, particularmente aqueles com um nível de antígeno prostático específico menor que $10 \mathrm{ng} / \mathrm{mL}$ e Gleason marca $3+3$ tumores (LITWIN; TAN, 2017). Cirurgia e radiação continuam sendo tratamentos curativos para doença localizada, mas apresentam efeitos adversos, como sintomas urinários e disfunção sexual, que podem afetar negativamente a qualidade de vida. Para doença metastática, a quimioterapia como tratamento inicial parece agora prolongar a sobrevida em comparação com a terapia de privação androgênica isolada. Novas vacinas, agentes hormonais terapêuticos e agentes de direcionamento ósseo demonstraram eficácia em homens com câncer de próstata metastático resistente à terapia hormonal tradicional (LITWIN; TAN, 2017).

A luta contra o câncer de próstata não termina nunca, pois as recomendações sobre prevenção e cura são constantes. Inclusive, a Biomatemática é uma ferramenta importante no auxílio ao combate do câncer de próstata. A modelagem matemática de processos relacionados ao desenvolvimento de tumores da próstata tem sido muito útil na obtenção de informações sobre as etapas da carcinogênese. As descobertas biológicas que levam a mudanças paradigmáticas na modelagem matemática, incluem o conceito de campo de cancerização, que coloca em questão a carcinogênese como elemento modelável. A compreensão de questões estruturais, como a vascularização de tumores sólidos, mecanismos de metástase, invasão e surgimento de resistência, trouxe uma perspectiva totalmente diferente sobre a modelagem do crescimento e progressão do câncer (KIMMEL, 2010). É claro que as informações baseadas por algum modelo matemático sempre devem ser verificadas por exames mais detalhados e por profissionais competentes, pois, o auxílio de um modelo matemático para este estudo não vem com o objetivo de substituir os diagnósticos atualmente empregados, porém deve servir como uma ferramenta de estudos sobre o crescimento de tumores da próstata. Sendo assim, dentro desse contexto, o objetivo do presente trabalho foi usar os métodos numéricos de diferenças finitas e Runge-Kutta para simular o crescimento de tumores malignos da próstata, usando a equação diferencial de Gompertz.

\section{DESENVOLVIMENTO}

\section{O câncer de próstata}

O câncer de próstata é o câncer mais comum entre os homens, mas muitas vezes pode ser tratado com sucesso. A cada ano novos casos da doença tem surgido no Brasil (BACELAR JÚNIOR et al., 2015).

\section{A Biomatemática}

$\mathrm{Na}$ oncologia, a biomatemática tem a finalidade de ajudar no combate ao câncer, combinando o conhecimento multidisciplinar de médicos e matemáticos. Muitos oncologistas, para não mencionar seus pacientes, podem pensar que não há lugar para a análise matemática no tratamento do câncer. Eles podem 
pensar que todas as decisões de tratamento são baseadas em fatores únicos que afetam pacientes individuais, sem conexão com outros pacientes e seus regimes de tratamento (MACKENZIE, 2004). Nesse caso, a biomatemática é uma combinação única de cuidados clínicos, pesquisa científica e conhecimentos matemáticos que melhoram a compreensão geral do desenvolvimento, crescimento, evolução e reação ao tratamento do câncer, ajudando os oncologistas a prever, controlar e combater a malignidade em escala global, aprimorando os padrões de cuidado baseados em evidências, e em um nível individual, personalizando o tratamento usando fatores quantificáveis de pacientes e doenças. Devido à sua natureza intrinsecamente interdisciplinar, a Biomatemática é de fundamental importância na prevenção e tratamento do câncer de próstata. Além de investigar questões de biologia do câncer, a Biomatemática usa dados de desfechos de grandes grupos de pacientes para criar modelos matemáticos preditivos, ou algoritmos, na esperança de gerar terapias efetivas independentes ou combinadas para pacientes individuais. Os algoritmos carregados com dados clínicos criam essencialmente um mapa de tratamento mais preciso para indivíduos que sofrem de cânceres semelhantes (MACKENZIE, 2004).

\section{Métodos numéricos}

A utilização de métodos numéricos em estudos sobre câncer tem como finalidade fornecer informações suficientes para a tomada de decisões. O objetivo é validar métodos matemáticos como uma poderosa ferramenta com a qual é possível modelar a natureza subjacente de sistemas caóticos e extrair conclusões úteis para problemas da vida real (JORDÃO; TAVARES, 2017). Os métodos numéricos ajuda a entender o metabolismo dos tumores malignos (MARKERT; VAZQUEZ, 2015). Isto é muito importante para se entender o crescimento de tumores malignos da próstata. O método das diferenças finitas é um método numérico bastante utilizado para resolver equações diferenciais. As diferenças finitas são apenas esquemas algébricos que se pode utilizado para aproximar derivadas. Os usos das diferenças finitas estão em qualquer disciplina onde se pode querer aproximar derivadas. As áreas de uso mencionadas abrangem a aplicação em muitos domínios, seja engenharia, ciência, negócios, medicina etc. Elas são uma ferramenta útil em muitas áreas, embora não sejam necessariamente a única abordagem para a aproximação de derivadas. Eles também não são as únicas ferramentas usadas para resolver equações diferenciais. Mas eles têm seu papel importante a desempenhar historicamente em muitas aplicações em que seu uso faz sentido. O método de Runge-Kutta é um método numérico muito utilizado para resolver equações diferenciais. Sendo assim, devido à fácil operacionalidade dos métodos numéricos de diferenças finitas e Runge-Kutta, eles podem ser utilizados para estudos de crescimento de tumores malignos da próstata.

\section{MATERIAL E MÉTODO}

\section{A equação diferencial de Gompertz}

Em 1825, Gompertz desenvolveu seu modelo para o crescimento populacional de uma determinada espécie qualquer, na qual, mostrou-se bastante eficaz quando comparado com os dados reais do crescimento de uma determinada população. Hoje, seu modelo é bastante utilizado em diversas áreas, em especial, no estudo da análise do crescimento de células tumorais (BOYCE; DEPRIMA, 2010).

A equação diferencial de Gompertz é dada pela Equação 1.

$$
\frac{d N(t)}{d t}=r N(t) \ln \left(\frac{k}{N(t)}\right)
$$


Onde:

- $\mathrm{N}(\mathrm{t})$ é a população tumoral de células em um determinado instante $\mathrm{t}$.

- R é a constante de crescimento intrínseco das células, com $r>0$.

- k é a capacidade de crescimento do tumor.

Para a resolução da Equação 1, é assumido que a população de células tumorais inicial é conhecida. Assim, a Equação 1 irá se tornar um problema de valor inicial, onde $N(0)=N_{0}$. Portanto, a Equação 1 transforma-se na Equação 2.

$$
\frac{d N(t)}{d t}=r N(t) \ln \left(\frac{k}{N(t)}\right), \operatorname{com} N(0)=N_{0}
$$

Resolvendo a Equação 2 obtemos a Equação 3. Então,

$$
\frac{d N(t)}{N(t) \ln \left(\frac{k}{N(t)}\right)}=r d t
$$

Integrando ambos os lados da Equação 3, vem,

$$
\int \frac{d N(t)}{N(t) \ln \left(\frac{k}{N(t)}\right)}=\int r d t
$$

Resolvendo a Equação 4, obtemos a Equação 5.

$$
N(t)=k e^{\ln \left(N_{0} / k\right) e^{-r t}}
$$

A Equação 5 representa o número de células tumorais em função do tempo baseado na equação diferencial de Gompertz. Através da Equação 5 foi possível simular o crescimento de tumores malignos na próstata.

Para um bom entendimento do comportamento do crescimento tumoral baseado na Equação de Gompertz, é necessário fazer uma análise da Equação 5, afim de entender suas características e aspectos de sua curva. Para isso, alguns pontos são importantes nesta análise como por exemplo: os pontos em que a Equação 5 é máxima e mínima; seus intervalos de crescimento e decrescimento; seus pontos de inflexão; a estabilidade do crescimento tumoral e também, um estudo de como se comporta a Equação 5, fazendo variar as constantes $r, N_{0}$ e $k$ em tempos $t$ fixos. Os pontos de máximo e mínimo de uma função (pontos críticos) são obtidos ao igualar a derivada desta função a zero. Fazendo isso para a Equação 5, e sabendo que sua derivada é justamente a Equação 1, os pontos críticos da curva do crescimento tumoral serão dado pela solução da Equação 6.

$$
r N(t) \ln \left(\frac{k}{N(t)}\right)=0
$$

A solução da Equação 6 é obtida para $N(t)=0$ e para $N(t)=k$, que são os possíveis pontos críticos da Equação 5. Para saber qual o valor do tempo correspondente a esses valores, basta substituí-los na Equação 5. Fazendo isso primeiramente para $N(t)=0$, obtém-se a Equação 7 .

$$
k e^{\ln \left(N_{0} / k\right) e^{-r t}}=0
$$


Como o lado esquerdo da Equação 7 é maior do que zero para qualquer $t$ pertencente aos reais, resta apenas verificar qual o ponto " $A^{\mathrm{w}}$ em que $\lim _{t \rightarrow A} N(t)=0$. Sabendo-se que $\lim _{x \rightarrow-\infty} e^{x}=0$, então obtemos a Equação 8.

$$
\lim _{t \rightarrow A} k e^{\ln \left(N_{0} / k\right) e^{-r t}}=0
$$

Para que a Equação 8 seja satisfeita, o expoente da base Neperiana tem que tender a $-\infty$ quando $t$ tender a $A$. Assim, obtemos a Equação 9.

$$
\lim _{t \rightarrow A} \ln \left(N_{0} / k\right) e^{-r t}=-\infty
$$
10.

Como o termo $\ln \left(N_{0} / k\right)$ é uma constante menor do que zero, a Equação 9 se reduz à Equação

$$
\lim _{t \rightarrow A} e^{-r t}=\infty
$$

Para satisfazer a Equação 10, o valor de $A$ deverá ser: $A=-\infty$. Portanto, para que $N(t) \rightarrow 0 \mathrm{e}$ consequentemente $d N(t) / d t \rightarrow 0, t$ deverá tender a $-\infty$. Como esse tempo é um tempo fora do intervalo estudado e sem significado físico, este ponto será descartado da análise. Agora, para encontrar o tempo correspondente a $N(t)=k$, basta seguir os mesmos passos feito anteriormente para a análise de $N(t)=0$. Fazendo essa análise, obtemos a Equação 11 .

$$
k e^{\ln \left(N_{0} / k\right) e^{-r t}}=k
$$

Simplificando a Equação 11, obtemos a Equação 12.

$$
e^{\ln \left(N_{0} / k\right) e^{-r t}}=1
$$

A Equação 12 será satisfeita quando o expoente da base Neperiana for igual a zero, como mostra a Equação 13.

$$
\ln \left(N_{0} / k\right) e^{-r t}=0
$$

Como o termo $\ln \left(N_{0} / k\right)$ da Equação 13 é uma constante já discutida anteriormente, a Equação 13 se reduzir à Equação 14.

$$
e^{-r t}=0
$$

Mas, a Equação 14 é diferente de zero para todo valor de $t$ pertencente aos reais. Então, mais uma vez, será necessário encontrar um valor " $B$ " que faça com que o lado esquerdo da Equação 14 tenda a zero Quando $t$ tender a B. Para isso, o expoente da base neperiana da Equação 14 deverá tender a $-\infty$, ou seja,

ou seja, como mostra a Equação 15.

$$
\lim _{t \rightarrow B}-r t=-\infty
$$


Sendo $r$ uma constante positiva, a solução da Equação 15 será $B=\infty$. Sendo assim, é concluído que $N(t) \rightarrow k$ quando $t \rightarrow \infty$. Essa observação é importante, pois ressalta o fato de que após um tempo muito grande, o crescimento tumoral tenderá a capacidade máxima. Como $d N(t) / d t \rightarrow 0$ quando $t \rightarrow \infty$, o ponto de carga máxima é também o ponto de estabilidade do crescimento tumoral, ou seja, o crescimento tumoral irá se estabilizar quando estiver em torno da sua carga máxima em suporte com todos os nutrientes que o tumor necessitará. Em resumo, se a população de células tumorais estiver abaixo do valor de $k$, ela tende a crescer em direção a esse valor. Se considerarmos, por algum motivo a população de células tumorais ultrapassou o valor que definimos para a capacidade de carga, ela também tenderá a esse valor, ou seja, como $k$ é o valor máximo que o tumor pode atingir, se por algum motivo $N(t)>k$, tem-se que, com o passar do tempo, $N(t) \rightarrow k$ (DOMINGUES, 2011). Teoricamente, o valor de $k$ somente seria alcançado após um tempo infinito, mas ele pode ser extrapolado a partir de dados experimentais (FIALHO, 1999). Para estudar os intervalos de crescimento e decrescimento da Equação 5, é notável que o crescimento tumoral é sempre maior do que zero e menor do que $k(0<N(t)<k)$, então, a partir disso é concluído que a Equação 1 será sempre maior do que zero $(d N(t) / d t>0)$. Portanto, a curva do crescimento tumoral não apresenta intervalos de decrescimento. Esse resultado mostra que, um determinado tumor encontrará no organismo, condições suficientes para crescer, sem interrupções, até atingir sua estabilidade. A aceleração do crescimento tumoral é definida como sendo a variação da taxa de crescimento ao longo do tempo, em termos matemáticos, esta seria a derivada segunda em relação ao tempo da Equação 5, ou simplesmente, a derivada em relação ao tempo da Equação 1.

Assim, derivando a Equação 1 em relação ao tempo, obtemos a Equação 16.

$$
\frac{d^{2} N(t)}{d t^{2}}=r\left[\ln \left(\frac{k}{N(t)}\right)-1\right] \frac{d N(t)}{d t}
$$

Substituindo o valor de $d N(t) / d t$ dado pela Equação 1 na Equação 16, obtém-se a aceleração do crescimento tumoral dado pela Equação 17.

$$
\frac{d^{2} N(t)}{d t^{2}}=r^{2} N(t) \ln \left(\frac{k}{N(t)}\right)\left[\ln \left(\frac{k}{N(t)}\right)-1\right]
$$

O ponto em que a aceleração do crescimento tumoral é nula, é justamente o ponto de inflexão do crescimento tumoral e também, o ponto em que a taxa do crescimento tumoral pode assumir valores extremos. Para a análise dos possíveis pontos que satisfaçam estas características, basta igualar o lado direito da Equação 17 a zero. Fazendo isto, obtemos a Equação 18.

$$
r^{2} N(t) \ln \left(\frac{k}{N(t)}\right)\left[\ln \left(\frac{k}{N(t)}\right)-1\right]=0
$$

A solução da Equação 18 resolvida para $N(t)=0, N(t)=k$ e para $N(t)=k / e$. Os dois primeiros pontos são justamente os pontos em que a Equação 1 é nula, cujo os cálculos já foram realizados. Como foi concluído que o primeiro ponto não tem sentido físico, a conclusão a respeito do segundo ponto é que, quando $t \rightarrow \infty, N(t) \rightarrow k$ e como foi visto, $d N(t) / d t \rightarrow 0$, assim como $d^{2} N(t) / d t^{2} \rightarrow 0$, ou seja, nesse ponto, a taxa de crescimento é zero e sua variação (aceleração) também é. Com isso, é possível afirmar que, com o passar do tempo, quando o crescimento tumoral atingir a sua capacidade máxima, a sua taxa de crescimento para de variar e assume um valor nulo, mantendo a 
população de células tumorais constante e estável. Agora, para verificar em que valor do tempo ocorre o último ponto de inflexão, basta substituir este na Equação 5, fazendo isto, obtemos a Equação 19.

$$
k e^{\ln \left(N_{0} / k\right) e^{-r t}}=\frac{k}{e}
$$

Simplificando a Equação 19, obtemos a Equação 20.

$$
e^{\ln \left(N_{0} / k\right) e^{-r t}}=e^{-1}
$$

ou seja, para satisfazer a Equação 20, os expoentes das bases Neperianas devem ser iguais, como mostra a Equação 21.

$$
\ln \left(N_{0} / k\right) e^{-r t}=-1
$$

Isolando a variável $t$ após algumas manipulações algébricas na Equação 21, é encontrado que o tempo em que ocorre o ponto de inflexão, ou seja, uma taxa de crescimento tumoral constante (aceleração nula), é dado pela Equação 22.

$$
t_{\text {inflexå }}=\frac{-1}{r} \ln \left(\frac{1}{\ln \left(k / N_{0}\right)}\right)
$$

Para analisar os intervalos de concavidade para cima ou para baixo da curva do crescimento tumoral, basta analisar quais são os intervalos em que a Equação 17 é maior ou menor do que zero. Esses intervalos representaram os intervalos em que a taxa do crescimento tumoral é crescente ou decrescente, mostrando como o tumor se desenvolve ao decorrer do tempo. De acordo com a Equação 17, esta possui apenas o termo "ln $(k / N(t))-1$ " que pode assumir valores maiores ou menores do que zero, já que todos os demais, sempre assumem valores positivos. Então, obtemos a Equação 23.

$$
\frac{d^{2} N(t)}{d t^{2}}>0 \Leftrightarrow \ln \left(\frac{k}{N(t)}\right)-1>0
$$

Assim, o intervalo em que a aceleração do crescimento tumoral assumirá valores positivos será dado no intervalo mostrado pela Equação 24.

$$
\ln \left(\frac{k}{N(t)}\right)>1
$$

A Equação 24 será satisfeita sempre que $\mathrm{N}(\mathrm{t})$ for dado pela Equação 25.

$$
N(t)<\frac{k}{e}
$$

Então, de acordo com a Equação 25, a taxa do crescimento tumoral será crescente sempre que a condição estabelecida por esta equação for satisfeita. Isso implica que, a curva do crescimento tumoral possui uma concavidade para cima para instantes de tempo que possua uma quantidade de células tumorais abaixo do representado pela Equação 22. Da mesma forma, é possível concluir que a aceleração do crescimento tumoral será menor do que zero para $\mathrm{N}(\mathrm{t})$ dado pela Equação 26.

$$
N(t)>\frac{k}{e}
$$


Então, a taxa do crescimento tumoral será decrescente nessa nova situação (Equação 26). Assim, como a concavidade da curva do crescimento tumoral será para baixo para instantes de tempo que possuam uma quantidade de células tumorais acima do representado pela Equação 22. Em resumo, as principais características matemáticas para o crescimento tumoral de uma célula cancerígena baseado no modelo de Gompertz são:

- A curva que representa o crescimento tumoral das células cancerígenas, $N(t)$, sempre apresentará um comportamento crescente em todo o intervalo.

- Esta mesma curva apresenta concavidade para cima para valores de $N(t)<k / e$ e concavidade para baixo para valores de $N(t)>k / e$.

- O ponto de inflexão $N(t)=k / e$ é dado para um tempo $t$ representado pela Equação 17 .

- $N(t) \rightarrow k, d N(t) / d t \rightarrow 0$ e $d^{2} N(t) / d t^{2} \rightarrow 0$ quando $t \rightarrow \infty$ significando que, ao passar de um longo tempo, o crescimento tumoral tenderá a se estabilizar em torno da capacidade de carga do tumor $k$.

Para obter essas soluções numéricas através do método das diferenças finitas e Runge-Kutta, utilizouse como ferramenta de apoio, o programa MATLAB que é um programa bastante eficaz para análises matemáticas e gerações de gráficos.

\section{RESULTADO E DISCUSSÃO}

\section{Solução numérica da Equação de Gompertz utilizando o método das diferenças finitas}

O método das diferenças finitas é um método de resolução de equações diferenciais que se baseia na aproximação de derivadas por diferenças finitas. A fórmula de aproximação obtém-se da série de Taylor da função derivada (Equação 27).

$$
f(x+h)=f(x)+f^{\prime}(x) h+\frac{f^{\prime \prime}(x) h^{2}}{2}+\frac{f^{\prime \prime \prime}(x) h^{3}}{6}+o\left(h^{4}\right)
$$

Para $f(x+h)=f(x)+f^{\prime}(x) h$, a derivada primeira poderá ser escrita pela Equação 28.

$$
f^{\prime}(x)=\frac{f(x+h)-f(x)}{h}
$$

A Equação 28 é conhecida como fórmula das diferenças progressivas. Através de um código desenvolvido na linguagem do MATLAB, a solução numérica da Equação 1, com os valores das constantes para a simulação do crescimento tumoral de um câncer de próstata e a solução analítica da mesma, foram comparadas para um incremento fixo $h=1$, como mostra a Figura 1. Na Tabela 1 encontram-se apresentado o Erro Relativo Médio do método das diferenças finitas com a solução analítica da Equação de Gompertz, para diferentes valores de $h$. 
Figura 1 - Comparação da solução da Equação de Gompertz para o crescimento de um tumor de próstata pelo método numérico das diferenças finitas, utilizando $h=1 \mathrm{e}$, pela forma analítica.

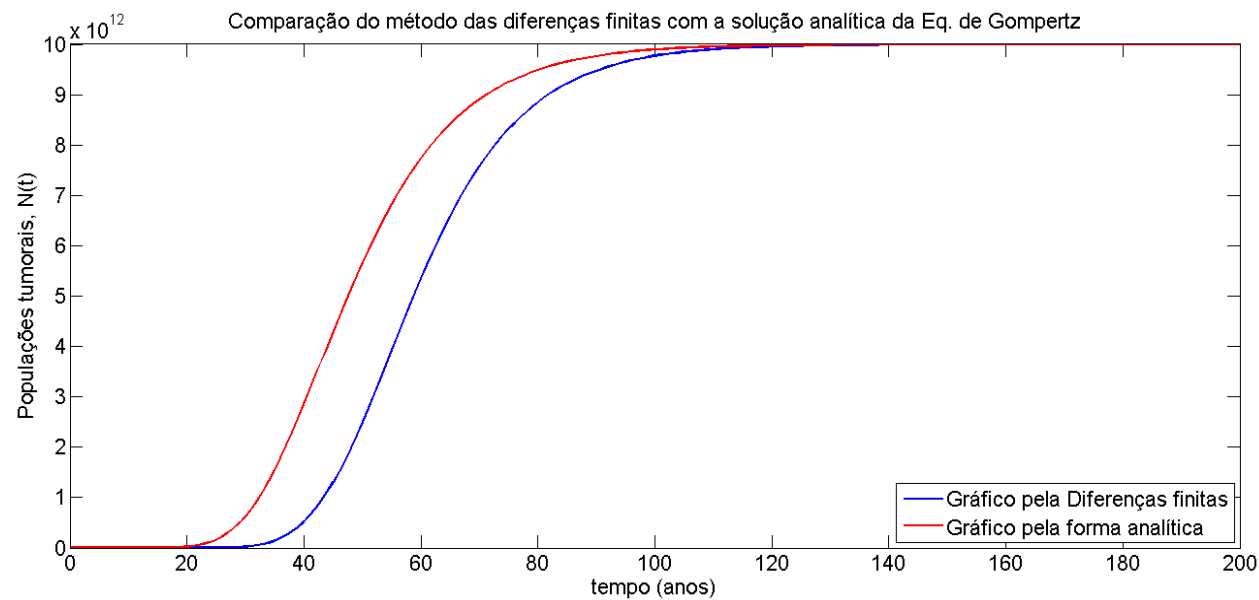

Tabela 1: Erro Relativo Médio em porcentagem do método numérico das diferenças finitas.

\begin{tabular}{c|c|c|c|c|c}
\hline $\begin{array}{c}\text { Incremento, } \\
h\end{array}$ & 0,001 & 0,01 & 0,1 & 1 & 10 \\
\hline $\begin{array}{c}\text { Erro Rel. } \\
\text { Médio (\%), } \\
\text { ERM(\%) }\end{array}$ & 0,104 & 1,014 & 7,958 & 26,345 & 60,002 \\
\hline
\end{tabular}

Como pode ser observado na Figura 1, para um $h=1$, o método das diferenças finitas diverge bastante da solução analítica, tendo com erro o valor de 26,345\%. Por outro lado, reduzindo o valor de $h$, o erro diminui significativamente, como mostra a Tabela 1. A Figura 2 mostra a representação gráfica para o incremento $h=0,1$. Observar-se na Figura 2 que, ao reduzir o valor do incremento, a solução numérica tornou-se mais próxima da solução analítica. De uma forma geral, o método das diferenças finitas só irá apresentar resultados satisfatórios para valores de $h$ muito pequenos, na ordem de pelo menos $10^{-3}$, pois para ordens de grandezas maiores, o valor do erro relativo já não pode ser desprezível. Sendo assim, a solução numérica da Equação 1 pelo método das diferenças finitas, é uma ferramenta bastante pratica e que, através dos erros obtidos, expressa a veracidade da solução analítica.

Figura 2 - Comparação da solução da Equação de Gompertz para o crescimento de um tumor de próstata pelo método numérico das diferenças finitas, utilizando $\boldsymbol{h}=\mathbf{0 , 1} \mathrm{e}$, pela forma analítica.

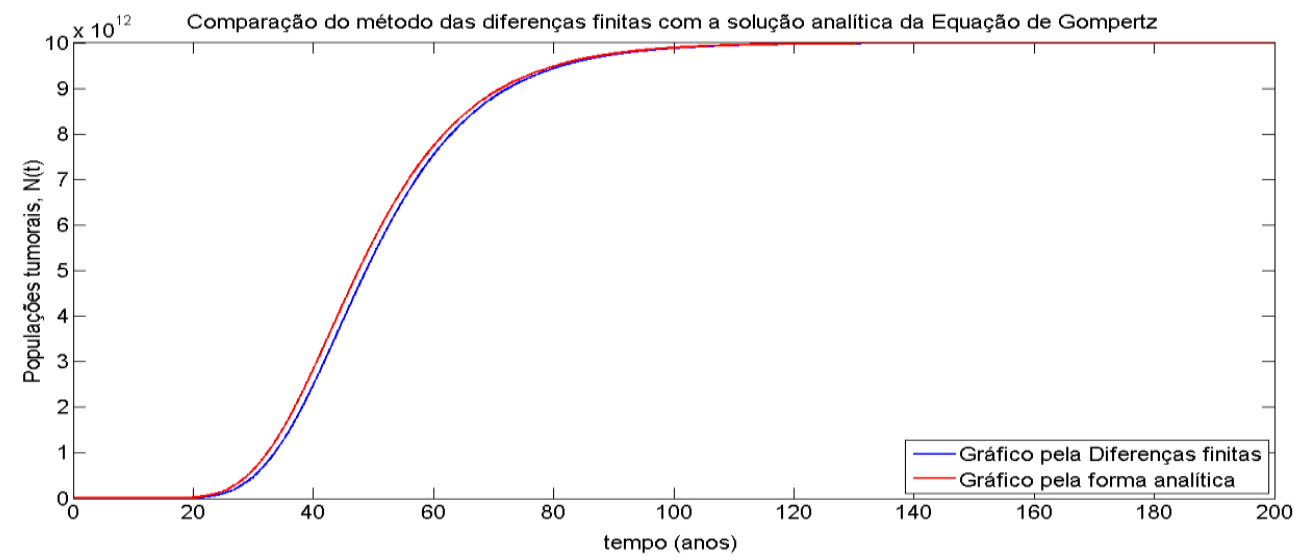




\section{Solução numérica da Equação de Gompertz utilizando o método de Runge-Kutta de ordem 4}

Cada método de Runge-Kutta consiste em comparar um polinômio de Taylor apropriado para eliminar o cálculo das derivadas. Fazendo-se várias avaliações da função a cada passo. Estes métodos podem ser construídos para qualquer ordem $\alpha$. Pelo polinômio de Taylor com resto, se uma função $y(x)$ tiver $k+1$ derivadas contínuas em um intervalo aberto contendo a e $x$, é possível escrever y (x) pela Equação 29.

$$
y(x)=y(a)+y^{s}(a) \frac{x-a}{1 !}+\cdots+y^{(k)}(a) \frac{(x-a)^{k}}{k !}+y^{(k+1)}(c) \frac{(x-a)^{k+1}}{(k+1) !}
$$

Onde $c$ é algum número entre $a$ e $x$.

Se substituir $a$ por $x_{n}$ e $x$ por $x_{n+1}=x_{n}+h$, a Equação 29 transforma-se na Equação 30.

$$
y\left(x_{n+1}\right)=y\left(x_{n}\right)+y^{\prime}\left(x_{n}\right) \frac{h}{1 !}+\cdots+y^{(k)}\left(x_{n}\right) \frac{(h)^{k}}{k !}+y^{(k+1)}(c) \frac{(h)^{k+1}}{(k+1) !}
$$

Para a obtenção do método de Runge-Kutta de ordem 4, basta fazer $k=4$ na Equação 30 para obter e Equação 31.

$$
y\left(x_{n+1}\right)=y\left(x_{n}\right)+y^{n}\left(x_{n}\right) \frac{h}{1 !}+y^{n !}\left(x_{n}\right) \frac{h^{2}}{2 !}+y^{n s}\left(x_{n}\right) \frac{h^{a}}{3 !}+y^{(4)}\left(x_{n}\right) \frac{h^{4}}{4 !}+y^{(5)}(c) \frac{h^{5}}{5 !}
$$

O procedimento de Runge-Kutta de quarta ordem consiste em encontrar constantes apropriadas, como mostra a Equação 32.

$$
y_{n+1}=y_{n}+a k_{1}+b k_{2}+c k_{3}+d k_{4}
$$

Considerando então a Equação 33.

$$
y\left(x_{n+1}\right)=y_{n}+h \varphi\left(x_{n}, y_{n}, h\right)
$$

Onde:

$k_{1}=h f\left(x_{n}, y_{n}\right)$

$k_{2}=h f\left(x_{n}+\alpha_{1} h, y_{n}+\beta_{1} k_{1}\right)$

$k_{3}=h f\left(x_{n}+\alpha_{2} h, y_{n}+\beta_{2} k_{1}+\beta_{3} k_{2}\right)$

$k_{4}=h f\left(x_{n}+\alpha_{3} h, y_{n}+\beta_{4} k_{1}+\beta_{5} k_{2}+\beta_{6} k_{3}\right)$

coincide com o polinômio de Taylor de grau quatro, como mostra a Equação 34 .

$$
p_{4}(x)=y(a)+y^{n}(a) \frac{(x-a)}{1 !}+y^{n g}(a) \frac{(x-a)^{z}}{2 !}+y^{n s}(a) \frac{(x-a)^{n}}{3 !}+y^{(4)}(a) \frac{(x-a)^{4}}{4 !}
$$

A solução da Equação 34 resulta em 11 Equações com 13 incógnitas, ou seja, possui infinitas soluções. O mais popular é dado pela Equação 35.

$$
y_{n+1}=y_{n}+\frac{1}{6}\left(k_{1}+2 k_{2}+2 k_{3}+k_{4}\right)
$$

com:

$$
\begin{aligned}
& k_{1}=h f\left(x_{n}, y_{n}\right) \\
& k_{2}=h f\left(x_{n}+\frac{1}{2} h, y_{n}+\frac{1}{2} k_{1}\right) \\
& k_{3}=h f\left(x_{n}+\frac{1}{2} h, y_{n}+\frac{1}{2} k_{2}\right) \\
& k_{4}=h f\left(x_{n}+h, y_{n}+k_{3}\right)
\end{aligned}
$$


Portanto, a Equação 35 é o método de Runge-Kutta de ordem quatro utilizada para se obter a solução numérica da Equação 1, utilizando as mesmas constantes destinadas a simular o crescimento de um tumor de próstata. Os resultados obtidos dos erros relativos médios estão apresentados na Tabela 2, para os mesmos incrementos $h$ da Tabela 1 . Foram usados os mesmos valores de h para fazer comparação entre os métodos. A Figura 3 mostra a comparação entre a solução analítica e a solução numérica do método de Runge-Kutta de ordem 4 para o incremento $h=1$.

Tabela 2: Erro Relativo Médio do método de Runge-Kutta de ordem 4.

\begin{tabular}{c|c|c|c|c|c}
\hline $\begin{array}{c}\text { Incremento, } \\
h\end{array}$ & 0,001 & 0,01 & 0,1 & 1 & 10 \\
\hline $\begin{array}{c}\text { Erro Rel. } \\
\text { Médio (\%), } \\
\operatorname{ERM}(\%)\end{array}$ & $9,354 \times 10^{-12}$ & $8,859 \times 10^{-8}$ & 0,000766 & 1,894 & 25,451 \\
\hline
\end{tabular}

A Figura 3 mostra a eficiência do método numérico de Runge-Kutta de ordem 4. O gráfico da solução numérica praticamente coincide com da solução analítica, sendo praticamente impossível fazer distinção entre ambas soluções. Assim, o método de Runge-Kutta de ordem 4 é um excelente método numérico para a solução da Equação 1. Por outro lado, o método das diferenças finitas não apresentou bons resultados para valores de $h$ acima de 0,01 , quando comparando com o método de Runge-Kutta de ordem 4. Assim, para adotar este método na solução da Equação 1, é necessário um valor de $h$ bastante pequeno. Apesar disso, o método das diferenças finitas apresentou resultados satisfatórios, aproximandose da solução analítica quando o valor do incremento foi de 0,01 .

Figura 3 - Comparação da solução da Equação de Gompertz para o crescimento de um tumor de próstata pela forma analítica e pelo método numérico de Runge-Kutta de ordem 4, utilizando $\boldsymbol{h}=\mathbf{1}$.

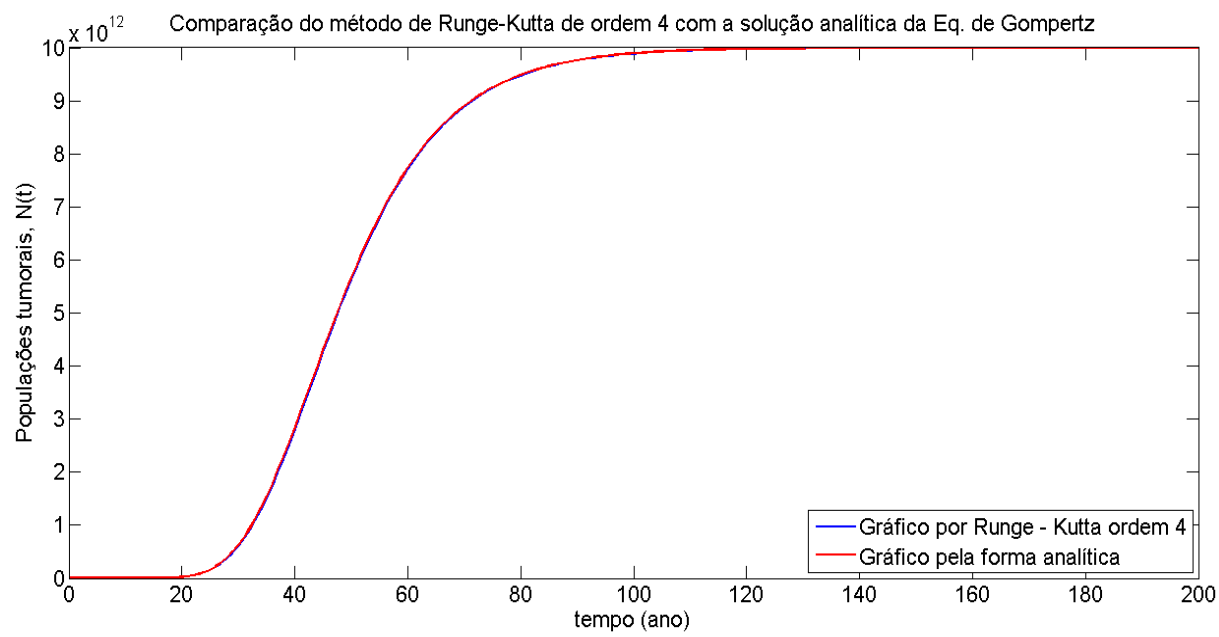

\section{CONCLUSÃO}

Os métodos numéricos das diferenças finitas e método de Runge-Kutta foram eficientes para calcular o crescimento de células tumorais na próstata usando a equação diferencial de Gompertz. $\mathrm{O}$ método de Runge-Kutta mostrou mais eficiente do que o método das diferenças finitas para valores de incremento maior do que 0,01 . 


\section{REFERÊNCIAS}

BACELAR JÚNIOR, A.J. et al. Câncer de próstata: métodos de diagnóstico, prevenção e tratamento. Brazilian Journal of Surgery and Clinical Research, v. 10, n.3, p. 40-46, 20015.

BOYCE, W.E., DIPRIMA, R.C. Equações diferenciais elementares e problemas de valores de contorno. 9rd ed. Rio de Janeiro:LTC;2010,607 p.

DOMINGUES, J.S. Análise do Modelo de Gompertz no crescimento de tumores sólidos e inserção de um fator de tratamento. Biomatemática IMECC - UNICAMP. MG, Pirapora, 2011.

FIALHO, F.S. Interpretação da curva de crescimento de Gompertz. Embrapa. SC, Concórdia, 1999.

JORDÃO, G., TAVARES J.N. Mathematical models in cancer therapy. Biosystems, v. 162, p. 12-23, 2017.

KIMMEL, M. Evolution and cancer: a mathematical biology approach. Biology Direct, v. 5, n.29, p. 1-3, 2010.

LITWIN, M.S., TAN, H.J. The diagnosis and treatment of prostate cancer: a review. JAMA, v. 317, n.24, p. 2532-2542, 2017.

MACKENZIE, D. Mathematical modeling and cancer. SIAM News, v.37, n.1, 2004.

MARKERT, E.K., VAZQUEZ, A. Mathematical models of cancer metabolism. Cancer Metabolism, v. 3, n.14, p. 1-13, 2015. 